\title{
A New Productivity Function and Stability Criterion in Chemical Vapor Transport Processes
}

\author{
K. KLOSSE* \\ Solid State Department, Physical Laboratory, University of Utrecht, Sorbonnelaan 4, \\ Utrecht, The Netherlands \\ Received October 8, 1974
}

\begin{abstract}
The crystal growth rate in a chemical vapor transport process using a closed system is analyzed on the basis of a one-dimensional configuration. A simplified model of vapor transport enables one to obtain a set of equations yielding the rates of reaction without a complete evaluation of the partial pressure gradients. This linear set comprises as many equations as independent chemical reactions. The effect of finite interface kinetics is formally taken into account. The efficiency of a one-reaction process is given by a function involving the mole fractions of the gaseous species and the stoichiometric coefficients in the formula equation. The features of such a productivity function are discussed. Maximum growth rate is achieved if the gaseous components are present in stoichiometric quantities. The concept of the productivity function is illustrated by chemical vapor transport systems involving binary and ternary gaseous phases. Proceeding from a two-phase source material, stability criteria that define stable one-phase and stable two-phase crystal growth are given. The kind of deposit may be changed by altering the amount of transporting agent. It is shown that limited interface kinetics favors a two-phase deposit.
\end{abstract}

\section{Introduction}

Chemical vapor transport is successfully used for the growing of crystals of relatively involatile materials. To facilitate the selcction of an efficient transport reaction, Schäfer has stated valuable rules supported by countless experiments (1). During the past several years numerous attempts have been made to provide a more accurate prediction of the crystal growth rate. I'ransport equations on the base of diffusion-limited vapor transport have been given by Mandel and his co-workers $(2-6)$, Arizumi and Nishinaga $(7,8)$, Faktor, Garrett and Heckingbottom (9), and undoubtedly by others. Due to their complexity, these procedures are generally less suited to preliminary evaluations of the efficiency in arbitrary reaction systems.

Alcock and Jeffes have considered the simplest vapor transport reaction, i.e., with

* Present address: Wolters-Noordhoff, P.O. Box 58, Groningen, The Netherlands.

Copyright 1975 by Academic Press, Inc. All rights of reproduction in any form reserved. Printed in Great Britain only two gaseous compounds present $(10,11)$. They have suggested that the efficiency may be related to a function involving the partial pressure of a given vapor species and the rate of change of this pressure with the equilibrium constant for the transport reaction. Since the fundamentals of a transport equation are lacking, it is not surprising that such an assumption leads them to a violation of Schäfer's rules.

Our present interest is to elucidate the characteristics of chemical vapor transport processes. We seek to replace Schäfer's qualitative rules by a quantitative understanding. The results should be easy to apply even in complex situations, so that a conceplual simplicity is pursued.

\section{Mass Transfer in the Gaseous Phase}

To calculate the crystal growth rate in a closed-tube process, the one-dimensional 
system shown in Fig. 1 will be used. The solid-gas interfaces are parallel planes distance $l$ apart. The chemical transport taking place from one temperature zone to the other, can be considered to consist of three steps:

(i) Transition of source material to the vapor phase by means of a chemical reaction and the formation of gaseous compounds of limited stability at $y=0$.

(ii) Mass transport in the gaseous phase from the source zone at $y=0$ to the seed site at $y=l$.

(iii) Decomposition of the vapor phase and deposition of a solid phase at the solidgas interface at $y=l$.

It is usually a reasonable assumption that (ii) will be the rate determining step. Then the pseudo-equilibrium chemical thermodynamics at the solid-gas interfaces are of major importancc. Concentration gradients in the gas phase caused by the temperature difference are the actual driving forces in mass transfer by diffusion. In the steady state these concentration gradients can be maintained by the continuous supply of material at $y=0$ and its removal from the gaseous phase at $y=l$.

Consider a chemical vapor transport system in which the material transport is based on one or more reactions of the general type

$$
0=\sum_{i} v_{r i}^{\prime} A_{i}(\mathrm{~s}, \mathrm{n})+\sum_{i} v_{r i} B_{i}(\mathrm{~g})
$$

where $v_{r i}^{\prime}$ and $v_{r i}$ are the stoichiometric coefficients of the solid or liquid compound $A_{i}$ and the gaseous compound $B_{t}$ in reaction $r$. The reactions take place at both interfaces
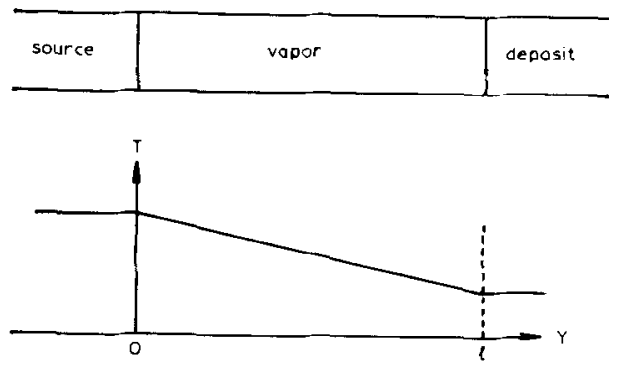

FIG. 1. Physical arrangement of a closed-tube process and schematic representation of the corresponding temperature distribution. in opposite directions. The equilibrium constant corresponding to Eq. (1) may be written

$$
K_{r}=\prod_{i} p_{i}^{y^{\prime l}}
$$

in which $p_{i}$ is the partial pressure of species $i$. In all cases gases will be treated as ideal.

Since the applied temperature difference is relatively small, the partial pressure differences are small as well. In other words, $\Delta p_{i} \ll p_{i}$ with $\Delta p_{t}=p_{i}(y=l)-p_{i}(y=0)$.

We will make use of $\Delta p_{i}^{\prime}$ only if we are dealing with parameters that are related to pressure gradients rather than to the partial pressures themselves. For example, material transfer by diffusion will be expressed in terms of $\Delta p_{i}{ }^{\prime}$.

We will trigger our theoretical analysis of the crystal growth rate by introducing the rate of reaction $\dot{\xi}_{r}$, one for each independent chemical reaction taking place at both interfaces. These rates of reaction are related to the flux of species $i$ in the gaseous phase, denoted $J_{i}$, by

$$
\sum_{r} v_{r i} \dot{\xi}_{r}=J_{i} O
$$

where $O$ is the cross-sectional area of the growth tube. Usually, these fluxes are calculated. However, note that the number of species generally exceeds the number of reactions greatly. Therefore, we will now present a sequence of steps leading to relationships from which the complete set of $\dot{\xi}_{\text {r }}$ values may be obtained straightforwardly. Hence, a considerable reduction of the number of simultaneous equations will be achieved by using rates of reaction instead of individual fluxes.

The assumption of uniform pressure gradients permits the flux to be written as

$$
J_{i}=-\frac{D}{l R T} \Delta p_{i}+\frac{v}{R T} p_{i}
$$

where $D$ is a diffusion coefficient; $R$ is the universal gas constant; $T$ is the absolute temperature; and $v$ is the laminar flow rate. We have assumed that $D, T, v$, and $p_{i}$ do not vary appreciably with position and have used averaged quantities. The diffusion term, $-D \Delta p_{i} / l R T$, describes the flux of species $i$ with respect to a general reference frame. In its 
turn, this reference frame is moving with a velocity $v$ with respect to the solid-gas interfaces at either side,

$$
v=\frac{R T}{P} \sum_{i} J_{i}
$$

where $P=\sum_{i} p_{i}$ represents the total pressure.

Let us say a few words on more accurate transport equations. Multicomponent diffusion coefficients like $D_{i j}$ may be used but nonlinear equations result. However, for most chemical transport reactions, these corrections are small compared to the uncertainties in the thermodynamic data. Generally, the use of multicomponent diffusion coefficients is a waste of computer time (12).

At high pressures, thermal convection must be taken into account. The ratio of the mass transfer by thermal convection and diffusion depends on the ampoule geometry and certain fluid dynamical parameters of the gaseous phase $(13,14)$. All we need do is adapt diffusion coefficient $D$ in Eq. (4). Such refinements, however, do not strike at the roots of the present theory.

In fact, we need not know the fluxes of the individual species explicitly for our analysis. From Eqs. (4) and (5), in the approximation $\Delta \ln p_{i}=\Delta p_{i} / p_{i}$, we obtain

$$
\Delta \ln p_{i}=\frac{l R T}{D}\left(\sum_{j} \frac{J_{j}}{P}-\frac{J_{i}}{P_{i}}\right) .
$$

In view of Eq. (3) and using mole fractions, $x_{i}=p_{i} / P$, Eq. (6) becomes

$$
\Delta \ln p_{i}=\frac{l R T}{O D P} \sum_{r}\left(\sum_{j} v_{r j}-\frac{v_{r t}}{x_{i}}\right) \dot{\xi}_{r} .
$$

We notice that the factor $I R T / O D P$ is independent of total pressure because of $D \propto P^{-1}$.

Till now, no use was made of the relationship between the set of $\Delta p_{i}$ values and the small shifts of the equilibrium constants caused by the temperature gradient:

$$
\left(1-\alpha_{r}\right) \Delta \ln K_{r}=\sum_{i} v_{r i} \Delta \ln p_{i}
$$

where $\quad \Delta \ln K_{r} \quad$ stands for $\ln K_{r}(y=l)-$ $\ln K_{r}(y=0)$ and $\alpha_{r}$ is a kinetic parameter. The effect of finite surface reaction rates can be accounted for by requiring that parameter $\alpha_{r}$ depend on the crystal growth rate, according to some law, in such a way that $0 \leqslant \alpha_{r} \leqslant 1$. Generally, if the growth rate vanishes, $\alpha_{r}$ vanishes as well. $\alpha_{r}=0$ is the situation of perfect equilibrium composition at both interfaces and it is the starting point for most calculations on diffusion controlled chemical vapor transport processes. $\alpha_{r}=1$ describes the situation of extreme kinetic hindrance at the interfaces in comparison with the mass transfer in the gaseous phase. In dissociative sublimation, for example, the gaseous phase and the growing crystal have the same composition. Since this situation does not require real diffusion, the material transfer is apparently not the slowest and rate determining step. As mentioned previously, we use average values for the partial pressures. That is why, mathematically, it does not matter whether the deviation from thermodynamical equilibrium comes from the kinetic hindrance at the source or at the seed site. This can be seen as follows. In both cases the average partial pressures are intermediate between the equilibrium values that belong to the upper and lower temperatures. It will be shown shortly that these partial pressures $V$ or the related mole fractions are the quantities that govern the characteristics of chemical vapor crystal growth.

Now, we are able to couple the rates of reaction and the thermodynamics of the transport process. Substitution of Eq. (7) into Eq. (8) leads to a set of simultaneous equations

$$
\sum_{s} a_{r s} \dot{\xi}_{s}=-\frac{O D P}{l R T}\left(1-\alpha_{r}\right) \Delta \ln K_{r},
$$

the coefficient $a_{r s}$ being given by

$$
a_{r s}=a_{s r}=\sum_{i} \frac{v_{r i} v_{s i}}{x_{i}}-\sum_{i} v_{r i} \sum_{i} v_{s i} .
$$

We see that the total number of equations equals the number of independent chemical reactions. Since both the right-hand side of Eq. (9) and the coefficients $a_{r s}$ are evaluated, easily, the rates of reaction can be derived without difficulty. 


\section{Productivity Function}

In the case of a one-reaction system the rate of reaction becomes [cf. Eq. (9)]:

$$
\dot{\xi}=-\frac{O D P}{l R T}(1-\alpha) \Delta \ln K F
$$

where $F$ is a productivity function defined by

$$
F=\left[\sum_{i} \frac{v_{i}^{2}}{x_{i}}-\left(\sum_{i} v_{i}\right)^{2}\right]^{-1}
$$

We see that the rate of reaction, and therefore, the crystal growth rate, are directly proportional to this productivity function. Once the stoichiometric coefficients are specified, the productivity function is entirely determined by the mole fractions $x$. It is demonstrated in Appendix $A$ that $F$ is always positive and takes its maximum value when $x_{i}=\hat{x}_{i}$,

$$
\hat{x}_{i}=\left|v_{i}\right| / \sum_{j}\left|v_{j}\right|
$$

which gives the maximum

$$
F_{\mathrm{m}}=\left[\left(\sum_{i}\left|v_{i}\right|\right)^{2}-\left(\sum_{i} v_{i}\right)^{2}\right]^{-1} .
$$

From Eq. (11), we see that the crystal growth rate decreases as the kinetic parameter $\alpha$ increases. We shall postpone our discussion of this subject to Section 6 .

For better understanding, we present an apparent contradiction between the theoretical productivity function and the experimental observations. For CdS, as an example of dissociative sublimation, we have

$$
2 \mathrm{CdS}(\mathrm{s}) \leftrightharpoons 2 \mathrm{Cd}(\mathrm{g})+\mathrm{S}_{2}(\mathrm{~g}) \text {. }
$$

If the gaseous phase consists merely of $\mathrm{Cd}$ and $\mathrm{S}_{2}$ in stoichiometric ratio, Eq. (13) is automatically satisfied and $F=F_{\mathrm{m}}$. Moreover, substituting the coefficients into Eq. (14) we find $F_{\mathrm{m}}=\infty$. In order to avoid an infinitely fast growth rate we have $\alpha=1$. For the gaseous phase, mass transfer is quite easy here; it is merely the flow of the gas as a whole, i.e., without diffusion.

Not all the sublimation experiments belong to this category. The presence of an inert gas or an additional reaction requires a certain diffusion process: $F_{\mathrm{m}} \neq \infty$.
Generally, as soon as gaseous products are present at both sides of the transport equation we have $F_{\mathrm{m}} \neq \infty$.

Lastly, we present an expression for the pressure gradient in a one-reaction process. Combining Eqs. (7), (11), and (12) we find

$$
\Delta \ln p_{i}=S_{i}(1-\alpha) \Delta \ln K
$$

where we have introduced

$$
\begin{gathered}
S_{i}=\left(\frac{v_{i}}{x_{i}}-\sum_{j} v_{j}\right) F, \\
S_{i}=-\frac{1}{2} \frac{\partial \ln F}{\partial v_{i}} .
\end{gathered}
$$

We call $S_{i}$ a stabilityu fnction because of its role in stability considerations, as will be shown presently.

\section{Binary Gaseous Phase}

Plots of the productivity function versus the equilibrium constant at fixed total pressure are given in Fig. 2 for the reaction

$$
X(\mathrm{~s}, \mathrm{l})+2 Z(\mathrm{~g}) \rightleftharpoons X Z_{2}(\mathrm{~g})
$$

and in Fig. 3 for

$$
4 X(\mathrm{~s}, 1)+Z_{4}(\mathrm{~g}) \rightleftharpoons 4 X Z(\mathrm{~g}) .
$$

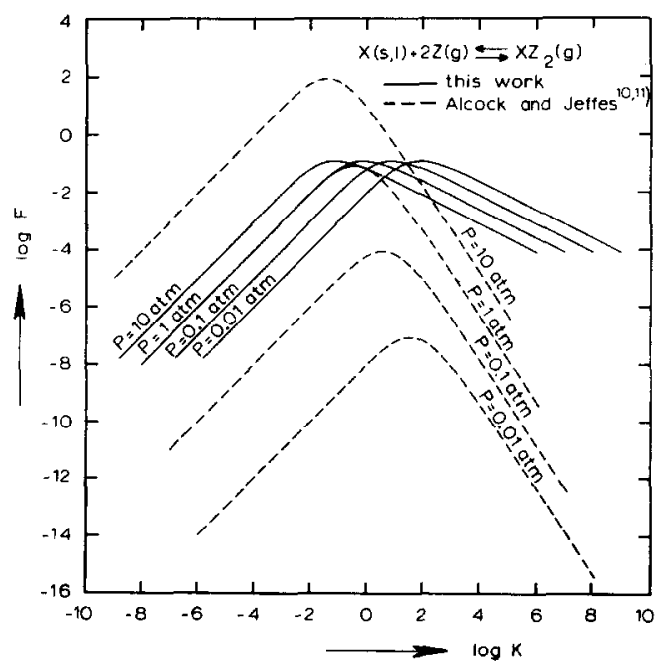

FIG. 2. Productivity function versus equilibrium constant at fixed total pressurc for $X(\mathrm{~s}, 1)+2 Z(\mathrm{~g}) \leftrightharpoons$ $X Z_{2}(\mathrm{~g})$. 


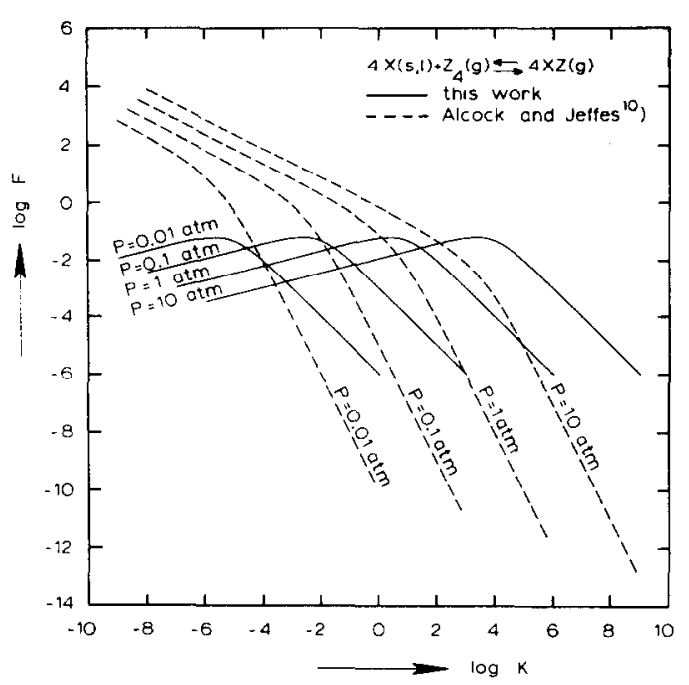

FIg. 3. Productivity function versus equilibrium constant at fixed total pressure for $4 X(\mathrm{~s}, 1)+Z_{4}(\mathrm{~g}) \leftrightharpoons$ $4 X Z(\mathrm{~g})$.

A change in total pressure displaces the curves only laterally. Generally, a factor of 10 in $P$ has the same effect as a factor of $10^{\Sigma_{t} v_{t}}$ in $K$. This is consistent with the fact that the mole fractions in a binary gaseous mixture and consequently the productivity function are unambiguously determined by $K / P^{\Sigma_{i} v_{l}}$ within the constraint of $\sum_{i} x_{i}=1$. Therefore, we have given in Fig. 4, the productivity function as a function of $K / P^{n-1}$ for the general formula reaction

$$
n X(\mathrm{~s}, \mathrm{l})+Z_{n}(\mathrm{~g}) \leftrightharpoons n X Z(\mathrm{~g}) .
$$

Before turning to more complex reactions, let us digress briefly to make a comment on a productivity function, introduced by Alcock and Jeffes $(10,11)$ for the same reactions as given in Eqs. (19) and (20). They defined a productivity function

$$
\text { P.F. }=p_{X z_{(n)}} \frac{d p_{X Z_{(n)}}}{d K}
$$

which was claimed to be a measure of the amount of material transported per unit increment of $K$. Their values are reproduced in Figs. 2 and 3. It will be clear that such a productivity function has very little to do with the experimental growth rate. In addition

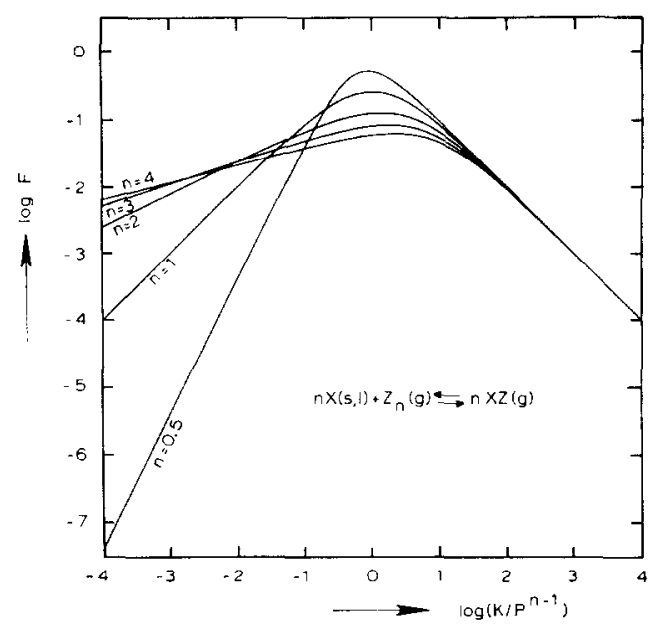

Fig. 4. Productivity function against $K / P^{n-1}$ for $n X(\mathrm{~s}, 1)+Z_{n}(\mathrm{~g}) \leftrightharpoons n X Z(\mathrm{~g})$

to the exaggerative influence of total pressure, the variation with the equilibrium position cannot be correct. In Fjg. 3, the more the reaction is shifted towards the extreme left, the greater the growth rate. Such a dependence contradicts one of the basic statements given by Schäfer: reactions for which the equilibrium position is extreme are unsuitable for chemical transport (I).

\section{Ternary Gaseous Phase}

Reactions involving three gaseous components can be written

$$
X_{n} Y_{m}(\mathrm{~s}, \mathrm{l})+Z_{n}(\mathrm{~g}) \leftrightharpoons n X Z(\mathrm{~g})+m Y(\mathrm{~g}) .
$$

The composition of the gaseous components and their stoichiometric coefficients may be chosen independently. Then, the composition of the nongaseous phase follows from conservation constraints. As an example, we present the transformation of a few reactions used in chemical vapor transport experiments

$$
\begin{gathered}
\mathrm{CdS}(\mathrm{s})+\mathrm{I}_{2}(\mathrm{~g}) \leftrightharpoons \mathrm{CdI}_{2}(\mathrm{~g})+\frac{1}{2} \mathrm{~S}_{2}(\mathrm{~g}), \quad(2 \\
2 \mathrm{GaAs}(\mathrm{s})+\mathrm{GaI}_{3}(\mathrm{~g}) \leftrightharpoons 3 \mathrm{GaI}(\mathrm{g})+\frac{1}{2} \mathrm{As}_{4}(\mathrm{~g})
\end{gathered}
$$

$$
\mathrm{Pt}(\mathrm{s})+2 \mathrm{CO}(\mathrm{g})+\mathrm{Br}_{2}(\mathrm{~g}) \leftrightharpoons \mathrm{Pt}(\mathrm{CO})_{2} \mathrm{Br}_{2}(\mathrm{~g}) \text {. }
$$


We use the following procedures (i) Take the correct stoichiometric coefficients: (ii) assign the parts of $Z_{n}, X Z$ and $Y$; (iii) subtract $Z$ as defined by $Z_{n}$ from $X Z$ to obtain $X$; and (iv) derive $X_{n} Y_{m}$. As a result, we obtain

$$
\begin{aligned}
& {[\mathrm{Cd}]_{1}\left[\mathrm{~S}_{2}\right]_{1 / 2}+\left[\mathrm{I}_{2}\right]_{1} \leftrightharpoons[\mathrm{Cd}]\left[\mathrm{I}_{2}\right]+\frac{1}{2}\left[\mathrm{~S}_{2}\right]} \\
& {\left[\mathrm{Ga}_{2 / 3}\right]_{3}\left[\mathrm{As}_{4}\right]_{1 / 2}+\left[\mathrm{Ga}_{1 / 3} \mathrm{I}\right]_{3}} \\
& \leftrightharpoons 3\left[\mathrm{Ga}_{2 / 3}\right]\left[\mathrm{Ga}_{1 / 3} \mathrm{I}\right]+\frac{1}{2}\left[\mathrm{As}_{4}\right] \\
& {\left[\mathrm{Pt}(\mathrm{CO})_{2}\right]_{1}[\mathrm{CO}]_{-2}+\left[\mathrm{Br}_{2}\right]_{1}} \\
& \leftrightharpoons\left[\mathrm{Pt}(\mathrm{CO})_{2}\right]\left[\mathrm{Br}_{2}\right]-2[\mathrm{CO}]
\end{aligned}
$$

This method may be complicated, but its disadvantage is fully compensated for by the resulting simplicity of the general mathematical problem.

Let us now return to the basic formula reaction [Eq. (23)]. The presence of three components in the gaseous phase leaves us two degrees-of-freedom as to the composition in terms of mole fractions. In addition to the ratio $K / P^{\Sigma_{t} v_{t}}$, we need a relation to specify the composition. We will discuss two different possibilities that cover most cases of practical interest.

First, we consider the one-phase source, with only one nongaseous compound, $X_{n} Y_{m}$, present. If we introduce a transporting agent $Z_{n}$, the gaseous products $X Z$ and $Y$ are formed in stoichiometric quantities according to Eq. (23), so that

$$
m x_{X Z}=n x_{Y}
$$

Again the productivity function will be uniquely determined by the ratio $K / P^{n+m-1}$ and the stoichiometric coefficients, $n$ and $m$. If $m=0$ we have the situation of a binary gaseous phase, as already discussed. The effect of a third gas component is demonstrated in Figs. 5 and 6 , where productivity functions versus $K / P^{n+m-1}$ are displayed for $n=1$ and $n=3$, respectively.

Second, we will discuss the consequences of a two-phase starting material. In addition to the component $X_{n} Y_{m}$, there is a second solid or liquid phase present at the source site, fixing the partial pressure of species $Y$

$$
X_{n} Y_{m}(\mathrm{~s}, 1) \leftrightharpoons X_{n} Y_{k}(\mathrm{~s}, 1)+(m-k) Y(\mathrm{~g}) \text {. }
$$

In Section 6 we will discuss the case in which $X_{n} Y_{k}(\mathrm{~s}, 1)$ is present in the seed zone. Therefore,

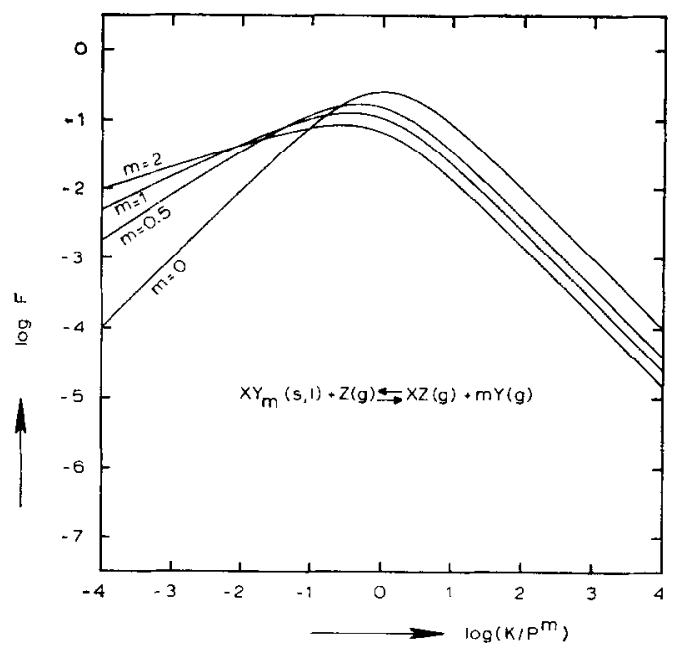

FIG. 5. Productivity function versus $K / P^{m}$ for $X Y_{m}(\mathrm{~s}, 1)+Z(\mathrm{~g}) \leftrightharpoons X Z(\mathrm{~g})+m Y(\mathrm{~g})$ using a one-phase source material.

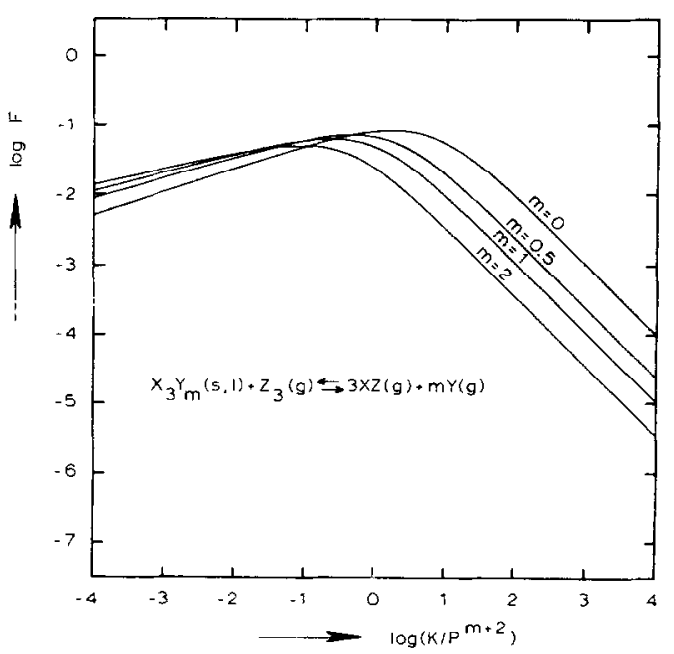

FIG. 6. Productivity function versus $K / P^{m+2}$ for $X_{3} Y_{m}(\mathrm{~s}, 1)+Z_{3}(\mathrm{~g}) \leftrightharpoons 3 X Z(\mathrm{~g})+m Y(\mathrm{~g})$ using a onephase source material.

we now bypass the question of an additional transport reaction involving $X_{n} Y_{k}$ analogous to Eq. (23).

Since Eq. (27) gives us a partial pressure and not a mole fraction we still need the total pressure. Then the productivity function will depend on $n, m, K, P$, and $p_{Y}$. Due to the extension of the number of parameters to be specified, a general representation in graphical 


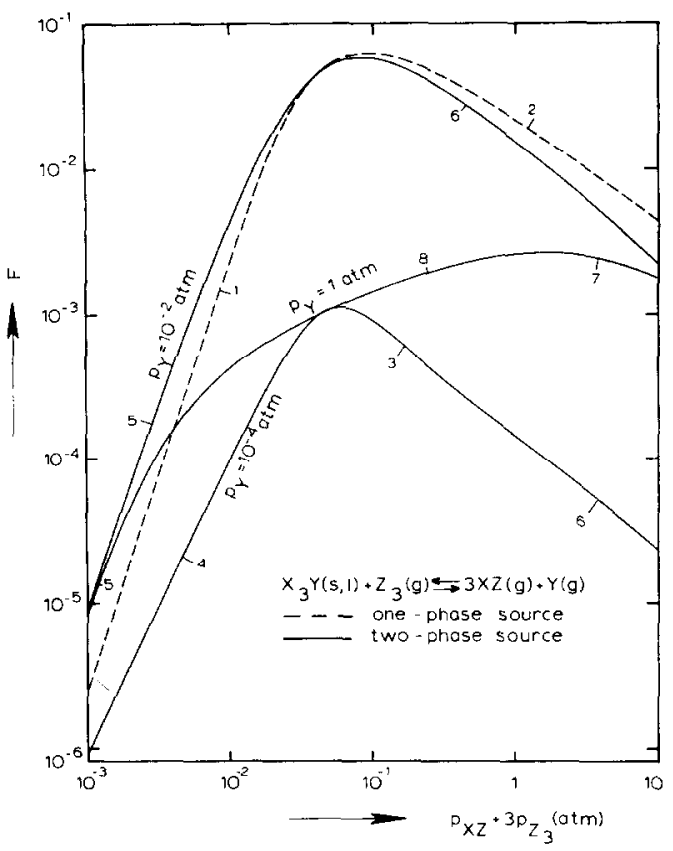

Fig. 7. Productivity function versus the amount of transport agent for $X_{3} Y(\mathrm{~s}, 1)+Z_{3}(\mathrm{~g}) \leftrightharpoons 3 X Z(\mathrm{~g})+$ $Y(\mathrm{~g}), K=10^{-4}$; relative importance of the gaseous components on the basis of the partial pressures according to: (1) $X Z, Y \gg Z_{3}$ and (2) $Z_{3} \gg X Z, Y$ for a one-phase source where $p_{X L}=3 p_{Y}$, and (3) $X Z \gg Z_{3} \gg$ $Y$, (4) $X Z \gg Y \gg Z_{3}$, (5) $Y \gg X Z \gg Z_{3}$, (6) $Z_{3} \gg X Z \gg Y$, (7) $Z_{3} \gg Y \gg X Z$, and (8) $Y \gg Z_{3} \gg X Z$ for a two-phase source where $p_{Y}$ is constant. form becomes impracticable. Hence, we illustrate the theory by choosing a special chemical vapor transport process, i.e.

$$
\begin{aligned}
& X_{3} Y(\mathrm{~s}, \mathrm{l})+Z_{3}(\mathrm{~g}) \leftrightharpoons 3 X Z(\mathrm{~g})+ Y(\mathrm{~g}), \\
& K=10^{-4} .
\end{aligned}
$$

Since the equilibrium constant $K$ is fixed, the productivity function can be given as a function of total pressure for diverse values of $p_{Y}$. However, in chemical transport processes it is often more convenient to use a parameter that is a measure of the amount of transporting agent. Therefore, we take instead of total pressure,

$$
z=p_{X Z}+n p_{Z_{n}}
$$

Figure 7 shows the productivity function as a function of $z$ for a one-phase source and a two-phase source, $p_{Y}=1,10^{-2}$, and $10^{-4} \mathrm{~atm}$. Note that the highest growth rate is achieved using a one-phase starting material. This is a general rule that follows immediately from the above extremum considerations.

To obtain a clearer understanding of the productivity function one has to be conscious of the dominating effect of the minority component. If, for example, $x_{Z_{n}} \ll x_{X Z}, x_{Y}$ the productivity function takes up the asymptotic value $F=x_{z_{n}}$. For this reason we have mentioned the relative importance of the components in Fig. 7. The corresponding asymptotic

\section{TABLE I}

\begin{tabular}{|c|c|}
\hline \multicolumn{2}{|r|}{ One-phase source, $p_{Y}=(m / n) p_{X Z}$} \\
\hline $\begin{array}{l}p_{X Z}, p_{Y} \gg p_{Z_{n}} \\
p_{Z_{n}} \gg p_{X Z}, p_{Y}\end{array}$ & $\begin{array}{l}F=z^{n+m-1} K^{-1} n^{1-m} m^{m}(n+m)^{-1} \\
F=z^{-(n+m-1) /(n+m)} K^{1 /(n+m)} n^{(m-1) /(n+m)} m^{-m /(n+m)}(n+m)^{-1}\end{array}$ \\
\hline \multicolumn{2}{|r|}{ Two-phase source, $p_{Y}=$ constant } \\
\hline $\begin{array}{l}p_{X Z} \gg p_{z_{n}} \gg p_{Y} \\
p_{X Z} \gg p_{Y} \gg p_{z_{n}} \\
p_{Y} \gg p_{X Z} \gg p_{z_{n}} \\
p_{z_{n}} \gg p_{X Z} \gg p_{Y} \\
p_{z_{n}} \gg p_{Y} \gg p_{X Z} \\
p_{Y} \gg p_{z_{n}} \gg p_{X Z}\end{array}$ & $\begin{array}{l}F=z^{-1} p_{Y} m^{-2} \\
F=z^{n-1} K^{-1} p_{Y}^{m} \\
F=z^{n} K^{-1} p_{Y}^{m-1} \\
F=z^{-1} p_{Y} n m^{-2} \\
F=z^{-1+1 / n} K^{1 / n} p_{Y}^{-m / n} n^{-1-1 / n} \\
F=z^{1 / n} K^{1 / n} p^{-1 / n / n} n^{2 \quad 1 / n}\end{array}$ \\
\hline
\end{tabular}

Asymptotic Expressions for the Productivity Function of the Reaction $X_{n} Y_{m}(\mathrm{~s}, 1)+Z_{n}(\mathrm{~g}) \leftrightharpoons n X 2(\mathrm{~g})+m Y(\mathrm{~g})$ 


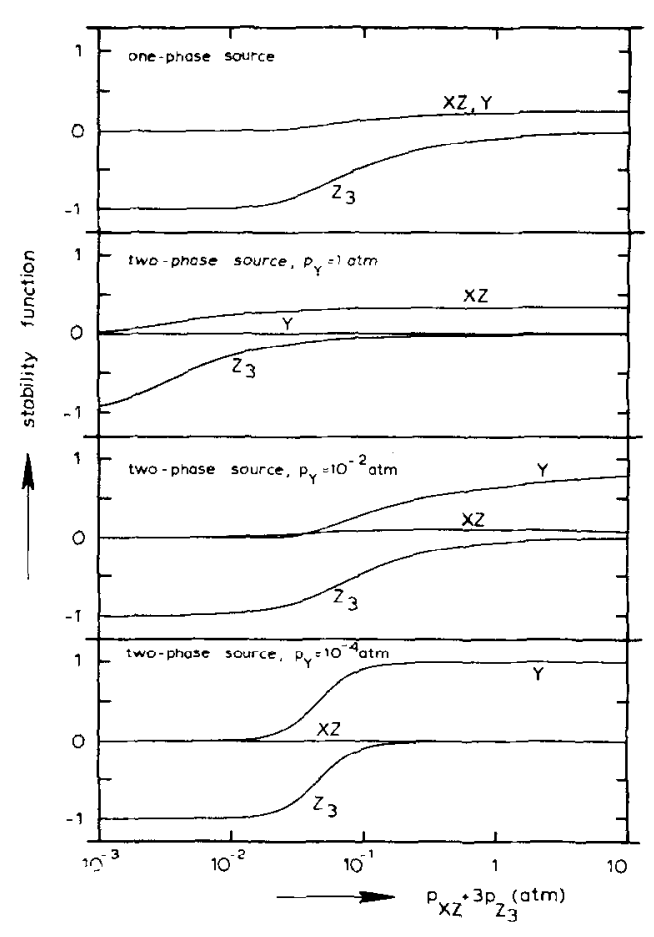

FIG. 8. Stability function versus the amount of transport agent for $X_{3} Y(\mathrm{~s}, \mathrm{l})+Z_{3}(\mathrm{~g}) \leftrightharpoons 3 X Z(\mathrm{~g})+$ $Y(\mathrm{~g}), K=10^{-4}$.

expressions of the productivity function are listed in Tablc I.

The importance of the minority component is demonstrated once more by the stability function $S_{i}$ being proportional to $\Delta \ln p_{i}$ [cf. Eq. (16)]. Figure 8 shows that $S_{i}$ differs appreciably from zero only if species $i$ is present in a minor concentration. In that case asymptotic values are $S_{i}=1 /(n+m)$ for $X Z$ and $Y$ using a one-phase source material and $S_{i}=1 / v_{i}$ in all other cases.

\section{Stability Considerations}

In Section 5 we met a growth reaction of the type

$$
X_{n} Y_{m}(\mathrm{~s}, 1)+Z_{n}(\mathrm{~g}) \leftrightharpoons n X Z(\mathrm{~g})+m Y(\mathrm{~g}) .
$$

In the presence of a two-phase source material the partial pressure of compound $Y$ was fixed according to

$$
X_{n} Y_{m}(\mathrm{~s}, 1) \leftrightharpoons X_{n} Y_{k}(\mathrm{~s}, \mathrm{l})+(m-k) Y .
$$

Now consider the possibility of a seed consisting of both $X_{n} Y_{m}$ and $X_{n} Y_{k}$. Thus, in addition to Eq. (30), we must use a second transport reaction involving $X_{n} Y_{k}$

$$
X_{n} Y_{k}(\mathrm{~s}, 1)+Z_{n}(\mathrm{~g}) \leftrightharpoons n X Z(\mathrm{~g})+k Y(\mathrm{~g}) .
$$

We will consider this problem more generally and put the reactions in the form

$$
\begin{aligned}
& 0=A_{1}(\mathrm{~s}, 1)+\sum_{i} v_{1 i} B_{i}(\mathrm{~g}), \\
& 0=A_{2}(\mathrm{~s}, 1)+\sum_{i} v_{2 i} B_{i}(\mathrm{~g}),
\end{aligned}
$$

where $v_{1 i}=v_{2 t}$ for $i \neq h$, so that species $h$ acts the part of compound $Y$. We must distinguish between the following situations.

\section{One-Phase Deposit}

As an example, $A_{2}$ is not present in the seed zone and the crystal growth is carried out exclusively by reaction (33a), so that

$$
a_{11} \dot{\xi}_{1}{ }^{I}=-\frac{Q D P}{l R T}\left(1-\alpha_{1}\right) \Delta \ln K_{1} .
$$

This gives us the general solution [cf. Eq. (11)]:

$$
\dot{\xi}_{r}^{I}=-\frac{O D P}{l R T}\left(1-\alpha_{r}\right) \Delta \ln K_{r} / a_{r r}
$$

where $r=1,2$. Since the coefficient $a_{r r}$ is the reciprocal of the productivity function it is always positive. If the applied temperature gradient permits crystal growth, $\Delta \ln K_{r}$ must be negative.

\section{Two-Phase Deposit}

The seed'consists of $A_{1}$ and $A_{2}$ and two equations must be satisfied

$$
a_{11} \dot{\xi}_{1}^{I I}+a_{12} \dot{\xi}_{2}^{I I}=-\frac{O D P}{l R T}\left(1-\alpha_{1}\right) \Delta \ln K_{1},
$$

$a_{21} \dot{\xi}_{2}^{I I}+a_{22} \dot{\xi}_{2}^{I I}=-\frac{O D P}{I R T}\left(1-\alpha_{2}\right) \Delta \ln K_{2}$.

Generally, we obtain

$$
\begin{aligned}
\dot{\xi}_{r} I= & -\frac{O D P}{l R T}\left[a_{s s}\left(1-\alpha_{r}\right) \Delta \ln K_{r}\right. \\
& \left.-a_{s r}\left(1-\alpha_{s}\right) \Delta \ln K_{s}\right] / a
\end{aligned}
$$


where $r=1, s=2$ or $r=2, s=1$, and $a=$ $a_{11} a_{22}-a_{12} a_{21}$. It is proved in Appendix B that $a$ is always positive.

First, let us discuss qualitatively the possible results. If $\dot{\xi}_{1}^{I I}>0$ and $\dot{\xi}_{2}^{I I}>0$, a deposit will result consisting of $A_{1}$ and $A_{2}$ in amounts proportional to the respective rates of reaction, i.e., two-phase crystal growth makes the condition

$$
\dot{\xi}_{1}^{I I}>0 \text { and } \dot{\xi}_{2}^{I I}>0 .
$$

On the other hand, if $\dot{\xi}_{1}^{I I}>0$ and $\dot{\xi}_{2}^{I I}<0, A_{2}$ moves towards the source zone up to depletion of the seed with respect to $A_{2}$. Then, a onereaction process takes over and a one-phase deposit of $A_{1}$ is formed. Thus, stable onephase growth of $A_{1}$ requires

$$
\dot{\xi}_{1}^{I I}>0 \text { and } \dot{\xi}_{2}^{I I}<0 .
$$

Note that the situation of both $\dot{\xi}_{1}^{I I}<0$ and $\dot{\xi}_{2}^{I I}<0$ cannot be reconciled with the premisses $\Delta \ln K_{1}<0$ and $\Delta \ln K_{2}<0$. To investigate stable one-phase growth of $A_{r}$, we may restrict ourselves to requiring that $\dot{\xi}_{s}^{I I}<0$. In view of Eq. (38) we get

$a_{r r}\left(1-\alpha_{s}\right) \Delta \ln K_{s}-a_{r s}\left(1-\alpha_{r}\right) \Delta \ln K_{r}>0$.

With the aid of the stability function [Eq. (17)] written in the form

$$
S_{r i}=\left(\frac{v_{r i}}{x_{i}}-\sum_{j} v_{r j}\right) / a_{r r}
$$

$a_{r s}[\mathrm{Eq} .(10)]$ may be related to $a_{r r}$ by

$$
a_{r s}=a_{r r}\left(1+\sum_{j}\left(v_{s i}-v_{r i}\right) S_{r i}\right) \text {. }
$$

Since $a_{r r}>0$ and $v_{r i}=v_{s i}$ for $i \neq h$, Eq. (41) may be written

$$
\begin{aligned}
\left(1-\alpha_{s}\right) \Delta \ln K_{s}- & {\left[1+\left(v_{s h}-v_{r h}\right) S_{r h}\right] } \\
& \times\left(1-\alpha_{r}\right) \Delta \ln K_{r}>0 .
\end{aligned}
$$

Starting from a two-phase material consisting of both $A_{r}$ and $A_{s}$, the growth of $A_{r}$ is stable provided that

$$
\left(v_{s h}-v_{r h}\right) S_{r h}>\frac{\left(1-\alpha_{s}\right) \Delta \ln K_{s}}{\left(1-\alpha_{r}\right) \Delta \ln K_{r}}-1 .
$$

Since for $\dot{\xi}_{s}^{I I}=0$ we havc $\alpha_{s}=0$, the demarcation between stable one-phase growth of $A_{r}$ and stable two-phase growth of $A_{r}+A_{s}$ is not affected by the kinetics of $A_{s}$. Thus, if $\alpha$ increases, the right-hand side of Eq. (45) also increases. This means that kinetic hindrance always favors the existence region of two-phase crystal growth.

Let us illustrate the stability criterion [Eq. (45)] on the basis of

$$
\begin{aligned}
X_{3} Y(\mathrm{~s}, \mathrm{l})+Z_{3}(\mathrm{~g}) & \leftrightharpoons 3 X Z(\mathrm{~g})+Y(\mathrm{~g}), \\
X_{3} Y_{3 / 2}(\mathrm{~s}, \mathrm{l})+Z_{3}(\mathrm{~g}) & \leftrightharpoons 3 X Z(\mathrm{~g})+\frac{3}{2} Y(\mathrm{~g}) .
\end{aligned}
$$

The equilibrium constants are taken $K_{1}=$ $10^{-4}, K_{2}=10^{-4}, 10^{-5}$, and $10^{-6}$, corresponding to $p_{Y}=1,10^{-2}$, and $10^{-4}$ atm, respectively. Figure 9 gives the kind of deposit as a function of $z=p_{x Z}+3 p_{z_{3}}$ and the ratio $\left(1-\alpha_{1}\right) \Delta \log K_{1} /\left(1-\alpha_{2}\right) \Delta \log K_{2}$. We see that the two regions of stable one-phase crystal growth are separated from each other by a zone of two-phase growth (shaded area).

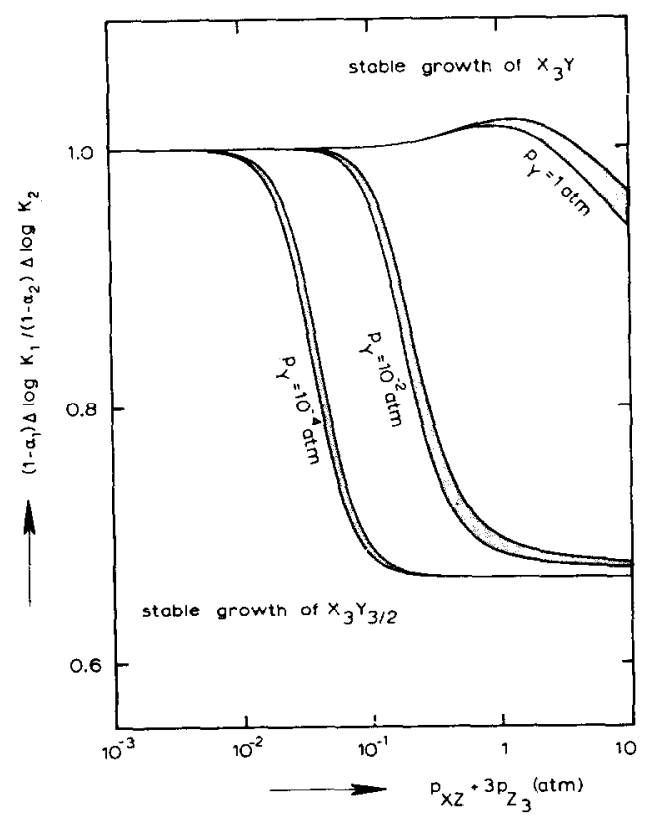

FIG. 9. Two-phase crystal growth (shaded area) separates the existence regions of stable one-phase growth in the case of a two-phase source and the reactions: (1) $X_{3} Y(\mathrm{~s}, \mathrm{l})+Z_{3}(\mathrm{~g}) \leftrightharpoons 3 X Z(\mathrm{~g})+Y(\mathrm{~g})$ and (2) $X_{3} Y_{3 / 2}(\mathrm{~s}, \mathrm{l})+Z_{3}(\mathrm{~g}) \leftrightharpoons 3 X Z(\mathrm{~g})+3 / 2 Y(\mathrm{~g}) \quad$ using $K_{1}=10^{4}$ and $K_{2}=10^{-4}\left(p_{Y}=1 \mathrm{~atm}\right), 10^{-5}\left(p_{X}=\right.$ $\left.10^{-2} \mathrm{~atm}\right), 10^{-6}\left(p_{Y}=10^{-4} \mathrm{~atm}\right)$. 
Growth rates are presented in the upper part of Fig. 10 for $K_{1}=10^{-4}, \Delta \log K_{1}=-0.4$, $K_{2}=10^{-6}, \quad \Delta \log K_{2}=-0.5, \quad p_{Y}=10^{-4}$ atm, and $\alpha_{1}=\alpha_{2}=0$. The ratio of $p_{Y}$ over the actual deposit and its equilibrium value over a two-phase seed are presented in the lower part of Fig. 10. The values are obtained from [cf. Eq. (31)]:

$$
\begin{aligned}
\Delta \ln p_{Y}= & {\left[\left(1-\alpha_{1}\right) \Delta \ln K_{1}\right.} \\
& \left.-\left(1-\alpha_{2}\right) \Delta \ln K_{2}\right] /\left(1-\frac{3}{2}\right)
\end{aligned}
$$

for a two-phase seed and from Eq. (17) for a one-reaction situation.

Up to this point, no explicit relation was used for the kinetic parameter $\alpha$. To demonstrate the effect of interface kinetics, we take a

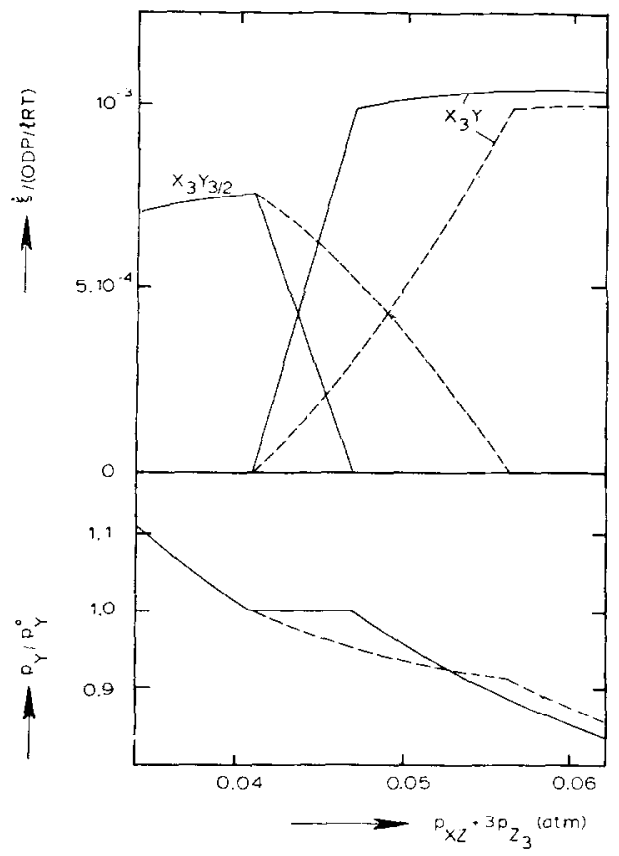

FIG. 10. Growth rates in units ODP/lRT (upper part) and the ratio of $p_{Y}$ over the actual deposit and its equilibrium value, $p_{Y}{ }^{\circ}$, over a two-phase seed (lower part) as a function of the amount of transport agent for the reactions: (1) $X_{3} Y(\mathrm{~s}, 1)+Z_{3}(\mathrm{~g}) \leftrightharpoons 3 X Z(\mathrm{~g})+$ $Y(\mathrm{~g})$ and $(2) X_{3} Y_{3 / 2}(\mathrm{~s}, \mathrm{l})+Z_{3}(\mathrm{~g}) \leftrightharpoons 3 X Z(\mathrm{~g})+3 / 2 Y(\mathrm{~g})$ using $K_{1}=10^{-4}, K_{2}=10^{-6}\left(p_{Y}=10^{-4} \mathrm{~atm}\right), \Delta \log K_{1}=$ $-0.4, \Delta \log K_{2}=-0.5, \alpha_{2}=0$. Solid lines correspond to the situation where kinetic hindrance of $X_{3} Y$ is absent, i.e., $\alpha_{1}=0$. Dashed lines are based on $\alpha_{1}=$ 0.05 at the point where the growth of $X_{3} Y+X_{3} Y_{3 / 2}$ passes into the growth of $X_{3} Y$. traditional dependence on crystal growth rate and partial pressures $V$ (hindrance of crystallization) according to

$$
\alpha \propto \xi / \prod_{i, v_{t}>0} p^{v_{t}}
$$

Thus, $\alpha$ vanishes if $\dot{\xi}$ approaches zero. The dashed curves in Fig. 10 correspond with $\alpha_{1}=$ 0.05 at the demarcation between the two-phase growth of $X_{3} Y+X_{3} Y_{3 / 2}$ and the one-phase growth of $X_{3} Y$, while $\alpha_{2}=0$, as above. Note the decrease of the growth rate of $X_{3} Y$ in both the two-phase and one-phase growth situations. Furthermore, there is considerable broadening of the two-phase region. We see that the growth of pure $X_{3} Y_{3 / 2}$ is completely insensitive to kinetic hindrance at the $X_{3} Y$ side, as expected. Finally, the partial pressure of $Y$ over a two-phase deposit is no longer a constant inasmuch as $\alpha_{1}$ in Eq. (47) varies according to Eq. (48).

\section{Summary and Conclusions}

The crystal growth rate for a chemical vapor transport process in a closed system has been discussed. To elaborate on this, the process was supposed to be a combination of simultaneous chemical reactions. A relatively simple model of vapor transport [Eq. (5)] leads to a set of equations that are linear in rates of reaction [Eq. (9)]; the coefficients depend on mole fractions and stoichiometric coefficients [Eq. (10)].

We have introduced a productivity function that is proportional to the crystal growth rate in a one-reaction system [Eq. (12)]. It has been shown that a function designed by Alcock and Jeffes $(10,11)$ falls in this regard. We have proved that maximum efficiency coincides with stoichiometry of the gaseous phase [Eqs. (13) and (14)]. General reaction types involving binary and ternary gaseous phases have been used to illustrate the theory. We have demonstrated the predominant effect of a minority gaseous component on crystal growth rate (Table I).

Using a two-phase starting material we have analyzed the conditions under which one-phase and two-phase deposits may be expected. A stability criterion has been formulated and discussed [Eq. (45)]. We have shown 
that limited interface kinetics decrease the growth rate and favors two-phase deposits. Moreover, the demarcation between onephase and two-phase growth is essentially changed by kinetics of the adjacent one-phase material.

\section{Appendix A}

For completeness we will discuss the extremum of the productivity function $F$ by considering

$$
1 / F=\sum_{i} \frac{v_{i}^{2}}{x_{i}}-\left(\sum_{i} v_{i}\right)^{2}
$$

where the mole fractions are restricted by $0<x_{i}<1$ and $\sum_{i} x_{i}=1$. The latter restraint can be used to eliminate, say, $x_{k}$. Thus we have

$$
\frac{\partial(1 / F)}{\partial x_{i}}=-\frac{v_{i}^{2}}{x_{i}^{2}}+\frac{v_{h}^{2}}{x_{h}^{2}}, \quad i \neq h .
$$

From the extremum condition $\partial(1 / F) / \partial x_{i}=0$ and the restriction $\sum_{i} x_{i}=1$, we obtain $\hat{x}_{i}$ as the value of $x_{i}$ for which both $1 / F$ and $F$ are extreme

$$
\hat{x}_{i}=\left|v_{i}\right| / \sum_{j}\left|v_{j}\right| \cdot
$$

This result leads to the extremum

$$
F_{\mathrm{m}}=1 /\left[\left(\sum_{i}\left|v_{i}\right|\right)^{2}-\left(\sum_{i} v_{i}\right)^{2}\right]
$$

which is always positive. The kind of extremum is found by expanding $1 / F$ in the neighborhood of $1 / F_{\mathrm{m}}$

$$
\begin{array}{r}
1 / F(x)=1 / F(\hat{x})+\frac{1}{2} \sum_{i, j \neq h}\left(x_{i}-\hat{x}_{i}\right) \\
\times\left(x_{j}-\hat{x}_{j}\right) \frac{\partial^{2}(1 / F(\hat{x}))}{\partial x_{i} \partial x_{j}} \\
=1 / F_{\mathrm{m}}+\sum_{i, j \neq h}\left(x_{i}-\hat{x}_{i}\right) \\
\times\left(x_{j}-\hat{x}_{j}\right)\left(\frac{v_{i}^{2}}{x_{i}^{3}} \delta_{i j}+\frac{v_{h}^{2}}{\hat{x}_{h}^{3}}\right) .
\end{array}
$$

Recalling that $\sum_{i \neq h}\left(x_{i}-\hat{x}_{i}\right)=-\left(x_{h}-\hat{x}_{h}\right)$, we obtain

$$
1 / F(x)=1 / F_{m}+\sum_{i} \frac{v_{i}^{2}}{\hat{x}_{i}^{3}}\left(x_{i}-\hat{x}_{i}\right)^{2}
$$

which describes the behavior of a minimum. As a conclusion, we state that the productivity function $F$ as a function of composition shows one maximum and is always positive.

\section{Appendix B}

The coefficients $a$ in Eq. (37) are given by Eq. (10)

$$
a_{r s}=\sum_{i} \frac{v_{r i} v_{s t}}{x_{i}}-\sum_{i} v_{r i} \sum_{i} v_{s i}
$$

which, since $v_{r i}=v_{s i}=v_{i}$ for $i \neq h$, may be rewritten

$$
\begin{aligned}
a_{r s}= & \sum_{i \neq h} \frac{v_{i}^{2}}{x_{i}}+\frac{v_{r h} v_{s h}}{x_{h}} \\
& -\left(\sum_{i \neq h} v_{i}+v_{r h}\right)\left(\sum_{i \neq h} v_{l}+v_{s h}\right) .
\end{aligned}
$$

Substitution into

$$
a=a_{11} a_{22}-a_{12} a_{21}
$$

gives

$$
a=\left(v_{r h}-v_{s h}\right)^{2}\left[\sum_{i \neq h} \frac{v_{i}^{2}}{x_{i}} \sum_{i \neq h} x_{i}-\left(\sum_{i \neq h} v_{i}\right)^{2}\right] / x_{h}
$$

The factor in brackets is just a reciprocal productivity function of a reaction with mole fraction $x_{i} / \sum_{i \neq h} x_{i}$, where species $h$ is missing. Therefore, as proven in Appendix A, $a$ must be definitely positive.

\section{Acknowledgments}

The valuable criticisms of Prof. J. H. van Santen and Dr. P. Ullersma during the course of this work are thankfully acknowledged. The author is indebted to Prof. G. Blasse for reading the manuscript. This work was performed as part of the research program of the "Stichting voor Fundamenteel Onderzoek der Materie (FOM)" and was made possible by financial support from the "Nederlandse Organisatie voor ZuiverWetenschappelijk Onderzoek (ZWO)."

\section{References}

1. H. SCHÄFER, "Chemische Transportreaktionen," Verlag Chemie, Weinheim, 1962.

2. G. Mandel, J. Phys. Chem. Solids 23, 587 (1962). 
3. R. F. Lever and G. Mandel, J. Phys. Chem. Solids 23, 599 (1962).

4. R. F. LeVER, J. Phys. Chem. Solids 26, 1629 (1965).

5. F. Jona AND G. Mandel, J. Chem. Phys. 38, 346 (1963).

6. F. Jona AND G. MANDEL, J. Phys. Chem. Solids 25, 187 (1964).

7. T. Arizumi and T. Nishinaga, Japan. J. Appl. Phys. 4, 165 (1965).

8. T. Arizumi and T. Nishinaga, Japan. J. Appl. Phys. 5, 21 (1966).
9. M. M. FaKtor, I. Garrett, AND R. Heckingвоттом, J. Crystal Growth 9, 3 (1971).

10. C. B. AlCock and J. H. E. JefFes, Trans. Inst. Mining Met. 76, C 246 (1967).

11. J. H. E. JEFFES, J. Crystal Growth, 3, 4, 13 (1968).

12. K. KLosse, Ph.D. thesis, State University Utrecht, 1973.

13. K. Klosse and P. Ullersma, J. Crystal Growth 18, 167 (1973).

14. C. Paorici, G. Attolini, C. Pelosi, and G. ZuCCALli, J. Crystal Growth 21, 227 (1974). 